\title{
Acute Toxicities and Prognosis of Elderly Patients with Nasopharyngeal Carcinoma After Intensity-Modulated Radiotherapy: Prediction with Nomogram
}

Cancer Management and Research

\author{
Yu Liang ${ }^{1,2}$ \\ Kai-hua Chen' \\ Jie Yang' \\ Jing Zhang' \\ Ru-rong Peng' \\ Song $\mathrm{Qu}^{\prime}$ \\ Ling $\mathrm{Li}^{\prime}$ \\ Xiao-dong Zhu (iD) ${ }^{1-4}$ \\ 'Affiliated Wuming Hospital of Guangxi \\ Medical University, Nanning, Guangxi, \\ People's Republic of China; ${ }^{2}$ Department \\ of Radiation Oncology, Guangxi Medical \\ University Cancer Hospital, Nanning, \\ Guangxi, People's Republic of China; ${ }^{3} \mathrm{Key}$ \\ Laboratory of Early Prevention and \\ Treatment for Regional High Frequency \\ Tumor (Guangxi Medical University), \\ Ministry of Education, Nanning, Guangxi, \\ People's Republic of China; ${ }^{4}$ Guangxi Key \\ Laboratory of Early Prevention and \\ Treatment for Regional High Frequency \\ Tumor, Guangxi Medical University, \\ Nanning, Guangxi, People's Republic of \\ China
}

Purpose: To explore acute toxicities and prognosis of elderly NPC patients after IMRT; to identify predictors regarding age, chemotherapy, comorbidities, nutrition status, and psychological condition; and to establish a nomogram for the prediction of prognosis.

Patients and Methods: Elderly NPC patients were divided into three groups (age of $60-65$, age of $66-70$, and age over 70) and were retrospectively analyzed. The acute toxicities, prognosis, and potential predictors were analyzed. Then, a nomogram for PFS was established, and the performance of nomogram was compared with the performance of TNM system.

Results: A total of 214 elderly patients $(214 / 1981,10.8 \%)$ were involved. Patients of Stage III and IV accounted for 73.4\%. The 3-year, 5-year PFS and OS were 77.9\%, 66.3\%, 79.3\% and $66.8 \%$, respectively. Elder patients had a worse prognosis $(P=0.002)$. The main cause of death remained in recurrence and metastasis; few died from comorbidities, and some died from nutrition status and psychological condition. Age $(\mathrm{HR}=1.10,95 \% \mathrm{CI}=1.05-1.15$, $P<0.001)$, ALB level $(\mathrm{HR}=0.93,95 \% \mathrm{CI}=0.88-0.99, P=0.019)$, and $\mathrm{T}$ stage $(\mathrm{HR}=1.85$, 95\% $\mathrm{CI}=1.10-3.13, P=0.022)$ were critical for PFS, but chemotherapy or comorbidities were not. Acute toxicities were mainly at or under grade II. $\mathrm{N}$ stage $(\mathrm{OR}=2.50,95 \%$ $\mathrm{CI}=1.28-4.88, P=0.007)$ and chemotherapy $(\mathrm{OR}=6.01,95 \% \mathrm{CI}=3.11-11.63, P<0.001)$ were risk factors for hematological toxicity; while age $(\mathrm{OR}=0.59,95 \% \mathrm{CI}=0.37-0.92, P=0.020)$ and chemotherapy $(\mathrm{OR}=225.14,95 \% \mathrm{CI}=61.91-818.64, P<0.001)$ influenced emesis; ALB $(\mathrm{OR}=1.11,95 \% \mathrm{CI}=1.04-1.19, P=0.002)$ affected mucositis. Comorbidities were not influential in acute toxicities. The nomogram for PFS ( $\mathrm{C}$-index $=0.682,95 \% \mathrm{CI}=0.617-0.747$ ) performed better than the TNM system $(\mathrm{C}$-index $=0.604,95 \% \mathrm{CI}=0.532-0.674, P<0.001)$.

Conclusion: Elderly NPC patients sustained poor prognosis. The easily applied nomogram is hopeful to benefit the clinical decision-making.

Keywords: cancer, geriatric, survival, outcome, side effect, model

\section{Introduction}

Nasopharyngeal Carcinoma (NPC) is epidemic and threatening in Southeast and Eastern Asia, ${ }^{1}$ and due to its unique anatomic location and radioactive sensitivity, the primary treatment is radiotherapy. NPC has a huge viability of onset age between 3-86 years old, but it is most prevalent among the population of 60-69year-olds. Therefore, elderly patients $(\geq 60)$ cover a certain proportion of NPC
Correspondence: Xiao-dong Zhu Affiliated Wuming Hospital of Guangxi Medical University, Nanning, Guangxi, People's Republic of China

Email zhuxdonggxmu@126.com 
patients. ${ }^{2}$ With the acceleration of aging progress worldwide, treatment of NPC in the elderly population has been a major health burden. Elderly patients have degeneration in body functions, poor psychological endurance, and a high number of comorbidities, therefore the management provides complex challenges for clinicians. However, the elderly patients have been under-represented in clinical trials, which results in a lacking of reliable evidencebased therapy. ${ }^{3,4}$ The survival of elderly patients is much worse than the general patients with a 5 -year overall survival as only about $50 \%{ }^{5,6}$ Many studies tried to work out a proper solution towards the treatment of elderly NPC patients, but due to the small sample size, ${ }^{7}$ outdated radiation technique, ${ }^{8,9}$ and old staging method, ${ }^{6,9}$ they restricted the applicability of their results. On the other hand, acute toxicities during the treatment play important roles in the clinical decision-making process, however little has been reported about what affects the acute toxicities. Age, chemotherapy, comorbidities, nutrition status, and psychological condition are supposed to have potential effects on both prognosis and acute toxicities, but little has been understood. Therefore, we conducted this study to explore the prognosis, acute toxicities, and their predictors of elderly patients with NPC after intensity-modulated radiotherapy (IMRT). Meanwhile, because nomograms are proved to be more reliable and efficient than the American Joint Committee on Cancer/Union for International Cancer Control (AJCC/UICC) Tumor-NodeMetastasis staging system (TNM system), we further developed a nomogram. To our knowledge, this is the first nomogram for the prognosis of elderly patients with NPC, and it is hopeful to promote proper and efficient clinical decision-making in the near aging future.

\section{Patients and Methods}

\section{Patients}

NPC patients admitted to our hospital during 2010-2016 were considered as potential participants. The inclusive criteria were as follows: patient who 1) was beyond 60 years old, 2) had histologic confirmation of nonkeratinizing nasopharyngeal carcinoma in our hospital, 3) had completement of IMRT. The exclusive criteria were the following: patient who 1) had distant metastasis at diagnosis; 2) had multiple primary carcinomas; and 3) had cancer treatment before. All patients were restaged using the 8th TNM system by two specialists of NPC independently.

\section{Treatments}

All patients underwent IMRT with or without chemotherapy. Regarding IMRT, the target volumes were delineated in accordance with the International Commission on Radiation Units and Measurements Reports 50 and 62. The prescribed doses were 70.06-75.00 Gy at 2.26-2.35 $\mathrm{Gy} /$ fraction to the planning target volume (PTV) derived from the gross tumor volume (GTV) of the primary tumor, 66.00-70.40 Gy to the PTV from the GTV of the nodal lesion, 60.00-64.00 Gy to the PTV from the clinical target volume with high risk (CTV1), and 54.00-57.60 Gy to the PTV of the low-risk clinical target volume (CTV2) in 30-33 fractions, the IMRT were delivered one fraction per day and 5 days per week. As for chemotherapy, patients with stage III/IV disease received concurrent chemoradiotherapy (CCRT) with or without sequential chemotherapy (induction chemotherapy (IC) and/or adjuvant chemotherapy (AC)), while patients with stage II disease received CCRT. The induction chemotherapy repeated every 3 weeks for $2-3$ cycles, TPF regimen (docetaxel $60 \mathrm{mg} / \mathrm{m}^{2}$ on day 1 , cisplatin $60 \mathrm{mg} / \mathrm{m}^{2}$ on day 1 , and 5 -fluorouracil $600 \mathrm{mg} / \mathrm{m}^{2}$ with continuous intravenous infusion during 120 hours) or PF regimen (cisplatin $80 \mathrm{mg} / \mathrm{m}^{2}$ on day $1-3$, and 5 -fluorouracil $750 \mathrm{mg} / \mathrm{m}^{2}$ with continuous intravenous infusion during 120 hours) was used. For concurrent chemotherapy, cisplatin (100 $\mathrm{mg} / \mathrm{m}^{2}$ on day $\left.1-3\right)$ was delivered every 3 weeks for 2-3 cycles. For adjuvant chemotherapy, PF regimen or TP regimen (docetaxel $75 \mathrm{mg} / \mathrm{m}^{2}$ on day 1 , cisplatin $75 \mathrm{mg} / \mathrm{m}^{2}$ on day 1) was applied every 3 weeks for $2-3$ cycles. If necessary, dose reductions would be applied at the discretion of doctors.

\section{Patient Assessment and Follow-Ups}

Data were collected for potential predictors, which enrolled baseline characters, disease history, laboratory examination (hemoglobin ( $\mathrm{Hb}$ ), albumin (ALB), lactate dehydrogenase level (LDH)), and treatment. According to the Common Terminology Criteria for Adverse Events (CTCAE) version 4.0 and the Radiation Morbidity Scoring Criteria of the Radiation Therapy Oncology Group, acute toxicities were graded. The end points were progress-free survival (PFS) and overall survival (OS). PFS was defined as a period from the first day of treatment to the day of recurrence, metastasis, death, or last visit. Overall survival was considered as a period from the first day of treatment to the day of death under any condition or the day of last visit. Patients were required to 
complete assessment every 2 months during the first year after treatment, every 3 months in the next 2 years and every 6 months thereafter.

\section{Statistical Analysis}

For non-survival data, statistical comparisons among groups were computed via variance analysis, chi-square test, or rank sum test if appropriate; Univariate analyses and multivariate analysis of acute toxicities were performed via logistic regression. For survival data, PFS and OS were calculated by Kaplan-Meier method and were compared by Log rank test among different subgroups. Univariate analyses and multivariate analysis of PFS were performed by the Cox proportional hazards model, and a backward stepwise selection method with Akaike information criterion was performed. ${ }^{10}$ Significant predictors in the multivariate analyses were used to establish the nomogram for PFS. Then the nomogram was compared with the 8th TNM system in regards of discrimination, calibration, and clinical usefulness. Discrimination was evaluated by concordance index (C-index); calibration was measured by calibration plots; and the clinical usefulness was depicted by decision curve. $^{11}$ The internal validation was carried out by bootstrapping.

A variable with a $P<0.10$ in univariable analysis was selected to enter multivariable analysis, while a two-side $P$-value $<0.05$ was considered as statistically significant on other occasions. Data analysis for nomograms was performed in $\mathrm{R}$ software (version 3.6), and other data were computed in SPSS (version 25.0).

Table I Charateristics of Patients and Tumor

\begin{tabular}{|c|c|c|c|c|c|}
\hline Characteristics (No./\%) & Total & $60 \leq n<65$ & $65 \leq n<70$ & $\geq 70$ & $P$-value \\
\hline Total & $214(100)$ & $127(59.3)$ & $51(23.8)$ & $36(16.8)$ & \\
\hline Sex & & & & & 0.218 \\
\hline Male & 165 (77.1) & $93(73.2)$ & $4 \mathrm{I}(80.4)$ & 31 (86.I) & \\
\hline Female & 49 (22.9) & $34(26.8)$ & $10(19.6)$ & $5(13.9)$ & \\
\hline $\mathrm{CCl}$ & & & & & 0.066 \\
\hline 0 & $135(63.1)$ & $85(66.9)$ & $33(64.7)$ & $17(47.2)$ & \\
\hline I & 64 (29.9) & $35(27.6)$ & $15(29.4)$ & 14 (38.9) & \\
\hline$\geq 2$ & $15(7.0)$ & $7(5.5)$ & $3(5.9)$ & $5(13.9)$ & \\
\hline BMI & & & & & $0.016 *$ \\
\hline$<18.5$ & $23(10.7)$ & $13(10.2)$ & $10(19.6)$ & $0(0.0)$ & \\
\hline $18.5-24$ & I44 (67.3) & $82(64.6)$ & $35(68.6)$ & $27(75.0)$ & \\
\hline$\geq 25$ & $47(22.0)$ & $32(25.2)$ & $6(11.8)$ & $9(25.0)$ & \\
\hline T stage & & & & & 0.891 \\
\hline $\mathrm{T} I \& 2$ & $95(44.4)$ & $58(45.7)$ & $22(43.1)$ & I5 (4I.7) & \\
\hline $\mathrm{T} 3 \& 4$ & $119(55.6)$ & $69(54.3)$ & $29(56.9)$ & $21(58.3)$ & \\
\hline $\mathrm{N}$ stage & & & & & 0.667 \\
\hline N0\&I & $112(52.3)$ & $67(52.8)$ & $28(54.9)$ & $17(47.2)$ & \\
\hline $\mathrm{N} 2 \& 3$ & $102(47.7)$ & $60(47.2)$ & $23(45.1)$ & $19(52.8)$ & \\
\hline Clinical stage & & & & & 0.930 \\
\hline I and II & $57(26.6)$ & $35(27.6)$ & $13(25.5)$ & $9(25.0)$ & \\
\hline III and IV & $157(73.4)$ & $92(72.4)$ & $38(74.5)$ & $27(75.0)$ & \\
\hline $\mathrm{ALB}($ mean, $\mathrm{SD})$ & $41.34,3.97$ & $41.76,3.90$ & $40.42,3.47$ & $41.14,4.70$ & 0.081 \\
\hline $\mathrm{LDH}$ (mean, SD) & $187.58,52.63$ & 178.70, 34.42 & $201.39,69.50$ & $199.33,70.59$ & 0.250 \\
\hline $\mathrm{Hb}$ (mean, SD) & $82.96,66.00$ & $81.12,67.41$ & $84.48,65.26$ & $87.32,63.52$ & 0.983 \\
\hline Treatment & & & & & $<0.001 * * *$ \\
\hline RT & $55(25.7)$ & $22(\mid 7.3)$ & $10(19.6)$ & $23(63.9)$ & \\
\hline RT+Chemotherapy & $159(74.3)$ & $105(82.7)$ & $4 \mid(80.4)$ & $13(36.1)$ & \\
\hline
\end{tabular}

Notes: $* P<0.05 ; * * * P<0.001$.

Abbreviations: $\mathrm{CCl}$, Charlson comorbidity index; BMI, body mass index; ALB, albumin; LDH, lactic dehydrogenase; Hb, hemoglobin; RT, radiotherapy. 


\section{Results}

\section{Baseline Characteristics}

As shown in Table 1, 214 patients were eligible, which accounted for $10.8 \%(214 / 1981)$ of the general NPC patients from the same period in our hospital. Age of the patients ranged between 60-86, with a median of 63.5; They were divided into three groups based on the age: $60 \leq \mathrm{n}<65$ group, $65 \leq \mathrm{n}<70$ group, and $\geq 70$ group. There were 127, 52, and 36 patients in the corresponding group, respectively. The male-to-female rate was about 3.4:1. One hundred and fifty-nine patients received chemotherapy (CCRT: 91 patients, IC+CCRT: 43 patients, CCRT+AC: 20 patients, IC+CCRT+AC: 1 patient, and IC only: 4 patients). In IC, $81 \%$ (39/48) received TPF regimen while others received PF; In AC, 61.9\% (13/21) used PF regimen while others used TP regimen. Cisplatin was replaced by other platinum complexes (nedaplatin or carboplatin) in five patients in IC and 16 patients in CCRT.

\section{Acute Toxicities}

As shown in Table 2, the common acute toxicities were recorded, including the hematological toxicity, emesis, mucositis, dermatitis, and weight loss. The acute toxicities were mostly at and under grade II, which indicated security during treatment. Meanwhile, comparisons were conducted among the three groups and it revealed statistically significant differences in hematological toxicity $(P=0.006)$

Table 2 Acute Toxicities and Prognosis

\begin{tabular}{|c|c|c|c|c|c|}
\hline Characteristics (No./\%) & Total & $60 \leq n<65$ & $65 \leq n<70$ & $\geq 70$ & $P$-value \\
\hline Total & 214 & 127 & 51 & 36 & \\
\hline Hematological toxicity & & & & & $0.006^{* *}$ \\
\hline 0 & $36(16.8)$ & $14(1 \mid .0)$ & $10(19.6)$ & $12(33.3)$ & \\
\hline 1 & $30(14.0)$ & $19(15.0)$ & $3(5.9)$ & $8(22.2)$ & \\
\hline 2 & $84(39.3)$ & $50(39.4)$ & $25(49.0)$ & $9(25.0)$ & \\
\hline 3 & $58(27.1)$ & $4 \mathrm{I}(32.3)$ & $10(19.6)$ & $7(19.4)$ & \\
\hline 4 & $6(2.8)$ & $3(2.4)$ & $3(5.9)$ & 0 & \\
\hline Emesis & & & & & $<0.00 I^{* * *}$ \\
\hline 0 & $54(25.6)$ & $20(\mid 5.7)$ & $10(19.6)$ & $24(66.7)$ & \\
\hline I & $78(37.0)$ & $49(38.6)$ & $23(45.1)$ & $6(16.7)$ & \\
\hline 2 & $64(30.3)$ & $48(37.8)$ & $14(27.5)$ & $5(13.9)$ & \\
\hline 3 & $15(7.1)$ & $10(7.9)$ & $4(7.8)$ & I (2.8) & \\
\hline 4 & 0 & 0 & 0 & 0 & \\
\hline Mucositis & & & & & $0.84 I$ \\
\hline 0 & I (0.5) & 0 & I (2.0) & 0 & \\
\hline I & $75(35.2)$ & $44(34.6)$ & $17(33.3)$ & $15(4 \mid .7)$ & \\
\hline 2 & $102(47.9)$ & $63(49.6)$ & $23(45.1)$ & $16(44.4)$ & \\
\hline 3 & $26(12.2)$ & $17(13.4)$ & $6(11.8)$ & $3(8.3)$ & \\
\hline 4 & $9(4.2)$ & $3(2.4)$ & $4(7.8)$ & $2(5.6)$ & \\
\hline Dermatitis & & & & & 0.798 \\
\hline 0 & $\mathrm{I}(0.5)$ & 0 & I (2.0) & 0 & \\
\hline 1 & $193(90.6)$ & $115(90.6)$ & $46(90.2)$ & $32(88.9)$ & \\
\hline 2 & $12(5.6)$ & $8(6.3)$ & $2(3.9)$ & $2(5.6)$ & \\
\hline 3 & $7(3.3)$ & $4(3.1)$ & $2(3.9)$ & I (2.8) & \\
\hline 4 & 0 & 0 & 0 & 0 & \\
\hline Weight loss (\%, median (range)) & $6.71(5.91-7.52)$ & $6.39(5.31-7.45)$ & $7.36(5.68-9.04)$ & $6.96(5.10-8.82)$ & 0.596 \\
\hline 3-year PFS & $77.90 \%$ & $83.30 \%$ & $75.60 \%$ & $62.80 \%$ & $0.002 * *$ \\
\hline 5-year PFS & $66.30 \%$ & $74.30 \%$ & $60.90 \%$ & $46.50 \%$ & $0.002^{* *}$ \\
\hline 3-year OS & $79.30 \%$ & $85.80 \%$ & $75.60 \%$ & $62.70 \%$ & $0.002^{* *}$ \\
\hline 5-year OS & $66.80 \%$ & $82.70 \%$ & $60.90 \%$ & $46.20 \%$ & $0.002^{* *}$ \\
\hline
\end{tabular}

Notes: $* * P<0.01$; $* * * P<0.001$.

Abbreviations: PFS, progress-free survival; OS, overall survival. 


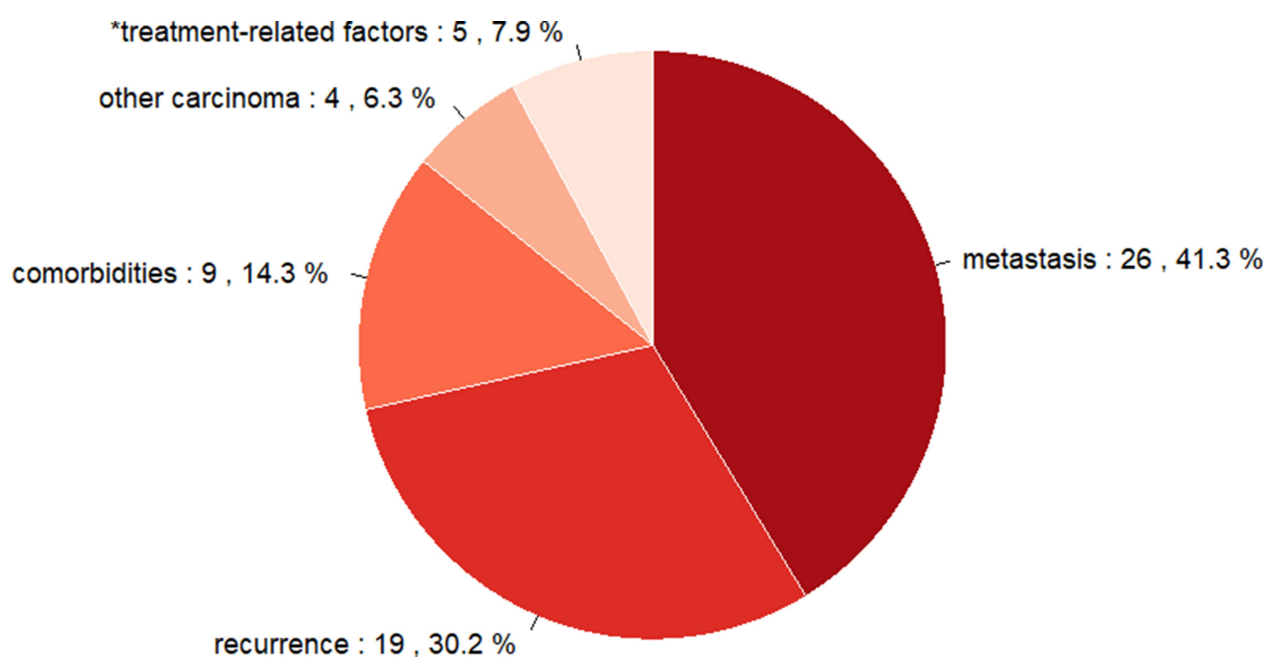

Figure I The cause of death in the elderly patients with NPC.

Notes: *Treatment-related factors: I) Suicide and apastia due to depressive mood caused by late toxicities: two patients; 2) Poor nutrient condition inducing by loss of appetite after treatment: two patients; 3) Severe epistaxis because of inappropriate care: one patient.

Abbreviation: NPC, nasopharyngeal carcinoma.

and emesis $(P<0.001)$, but no statistically significant difference in the other three acute toxicities.

\section{Survival Outcome}

The follow-up time ranged from 2-98 months, with a median of 44 months. Sixty-three patients died during this time, and the cause of death was pictured in Figure 1: most of them died from recurrence or metastatic $(71.43 \%)$, nine patients died from comorbidities previously existed, and, unexpectedly, five patents lost lives from avoidable treatment-related factors caused by poor nutrition status and psychological condition, such as suicide and apastia.

The PFS and OS were depicted in Table 2. The total 3 -year and 5-year PFS was $77.9 \%$ and $66.3 \%$, respectively, and the total 3-year and 5-year OS was $83.3 \%$ and $74.3 \%$, respectively. Both the 3-year, 5-year PFS and OS were worse in the elder patients, with a significant difference detected among three groups (all $P=0.002$ ). Subgroup analyses based on clinical stage was also conducted, and results are shown in Figure 2.

\section{Univariate and Multivariate Analysis of Acute Toxicities}

We conducted univariate and multivariate analysis in common acute toxicities (Table 3), and found that $\mathrm{N}$ stage $(\mathrm{OR}=2.50,95 \% \mathrm{CI}=1.28-4.88, P=0.007)$ and chemotherapy $(\mathrm{OR}=6.01,95 \% \mathrm{CI}=3.11-11.63, P<0.001)$ were statistics significant for hematological toxicity; we also found that age $(\mathrm{OR}=0.59,95 \% \mathrm{CI}=0.37-0.92, P=0.020)$ and chemotherapy $\quad(\mathrm{OR}=225.14, \quad 95 \% \quad \mathrm{CI}=61.91-818.64$, $P<0.001)$ were statistically significant for emesis, while ALB (OR=1.11, 95\% CI=1.04-1.19, $P=0.002)$ affected mucositis and no factors were proved to be significant for dermatitis.

\section{Univariate and Multivariate Analysis of PFS}

As shown in Table 4, univariate analysis indicated that the potential factors of PFS were age, sex, LDH, ALB, T stage, $\mathrm{N}$ stage, and clinical stage, however $\mathrm{CCI}, \mathrm{BMI}, \mathrm{Hb}$, and chemotherapy were not of statistical significance. Multivariate analysis indicated that Age $(\mathrm{HR}=1.10$, 95\% CI $=1.05-1.15, \quad P<0.001), \quad$ ALB $\quad(\mathrm{HR}=1.11, \quad 95 \%$ $\mathrm{CI}=1.04-1.19, \quad P=0.002)$, and $\mathrm{T}$ stage $(\mathrm{HR}=1.85,95 \%$ $\mathrm{CI}=1.10-3.13, P=0.022)$ were statistically significant in PFS.

\section{The Nomogram of PFS}

Based on statistical significance in the multivariate analysis of PFS and clinical importance, the nomogram was constructed by variables including sex, age, ALB, T stage, and $\mathrm{N}$ stage (Figure 3). In the nomogram, the total point was oneto-one corresponded with the 3-year PFS, so that clinicians can sum up points of each variable and then easily estimate the survival outcome. In our nomogram, the C-index was $0.682(95 \% \mathrm{CI}=0.617-0.747)$, and it had better performance than the 8th TNM system (C-index $=0.604, \quad 95 \%$ $\mathrm{CI}=0.532-0.674, P<0.001)$. Figure 4 demonstrated the calibration of the nomogram and the TNM system; Figure 5 was 

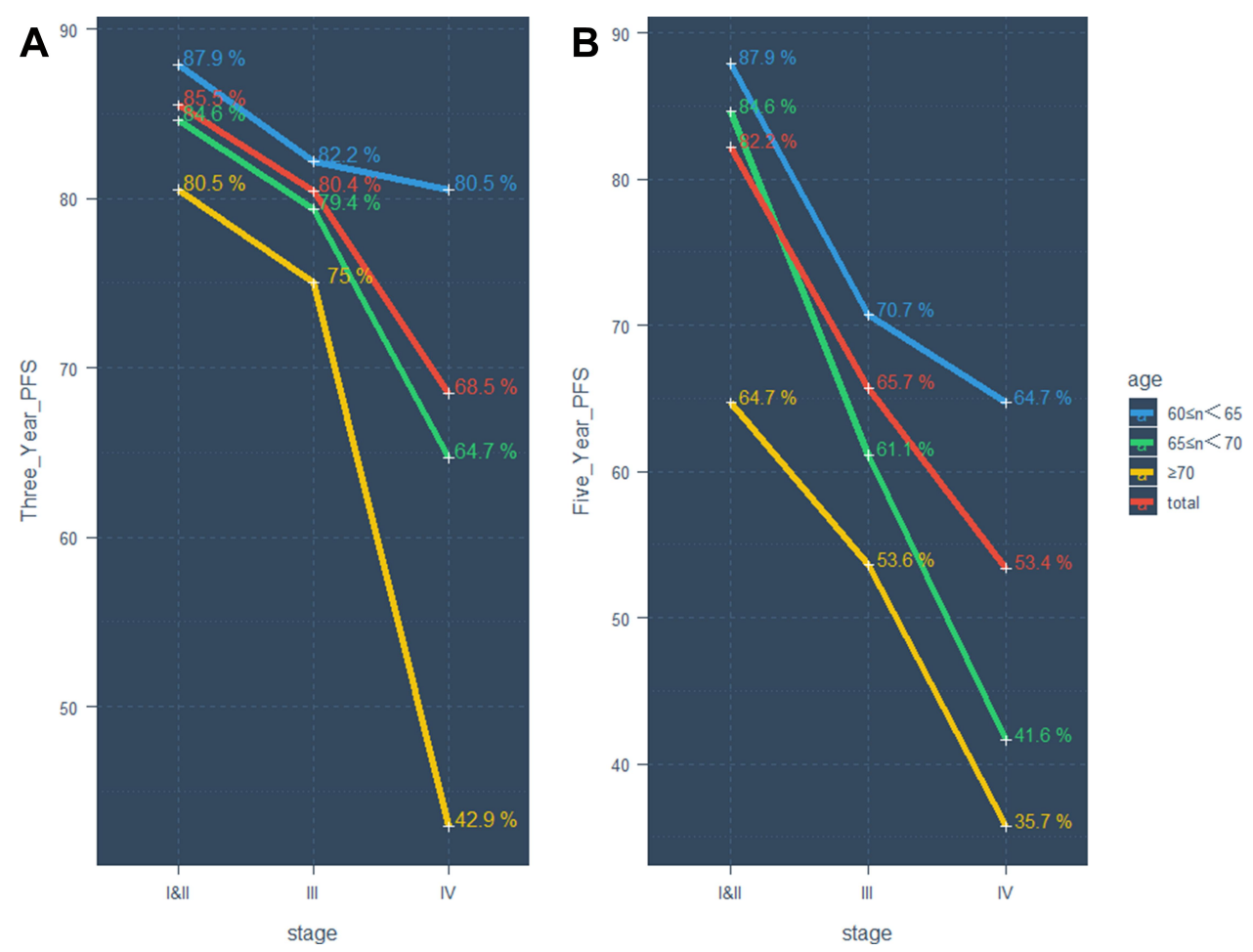

Figure 2 3-year PFS (A) and 5-year PFS (B) for the elderly patients with NPC of different ages and stages.

Abbreviations: PFS, progress-free survival; NPC, nasopharyngeal carcinoma.

the decision curve, and it showed that the nomogram did better in clinical usefulness than the TNM system.

\section{Discussion}

To our knowledge, this is the first nomogram for the prognosis of elderly patients with NPC. The long-term survival outcome is unsatisfied in the elderly patients and they were often ruled out by most of the clinical trials. Therefore, clinical decision-making for them is in great need of evidence-based support. Several studies analyzed the elderly NPC patients, however, their results were under obvious limitation because of the small size, usage of outdated radiation technique and the old TNM system. Meanwhile, little is known about acute toxicities and what their affective factors are. Age, chemotherapy, comorbidities, nutrition status, and psychological condition are supposed to play important roles in both prognosis and acute toxicities, but little is understood. Therefore, in this study, we explored the prognosis, acute toxicities, and their predictors of elderly patients with NPC. The results showed that the total 3-year, 5-year PFS and OS was $77.9 \%, 66.3 \%, 79.3 \%$, and $66.8 \%$, respectively. Patients were divided into three groups by their age (60-65, 65-70, and $\geq 70$ groups), and it indicated that the older a patient is, the worse the prognosis is; The main causes of death were still cancer recurrence and metastasis, a few patients died from comorbidities, and some of them died from avoidable treatment-related factors caused by poor nutrition status and psychological condition, such as suicide and apastia. The acute toxicities during treatment were mainly at or under grade II and thus tolerable in most cases; multivariate analyses indicated that $\mathrm{N}$ stage and chemotherapy played important roles in hematological toxicity; Age and chemotherapy influenced the emesis; and ALB affected the grade of mucositis. The multivariate analysis for PFS showed that age, T stage, and ALB were critical. Based on the results of the multivariable analysis of PFS, the nomogram was established and was proved to be more reliable than the TNM system. Comorbidities quantified by CCI were not a significant factor of neither PFS nor acute toxicities.

Along with the enhancement of radiation technique and the improvement of the regimen and sequence of chemotherapy, it has made significant progress in the longterm survival for general NPC patients. ${ }^{1,12}$ However, the elderly patients bear poor survival outcomes: the OS of the elderly NPC patients was reported to be only $51.1 \%$ from a study of $2007 ;^{13}$ Gao et $\mathrm{al}^{8}$ declared that the survival 
Table 3 Univariate and Multivariate Analysis of Acute Toxicities

\begin{tabular}{|c|c|c|c|c|c|c|c|c|}
\hline \multirow{3}{*}{$\begin{array}{l}- \\
\text { Characteristics }\end{array}$} & \multirow{2}{*}{\multicolumn{2}{|c|}{$\begin{array}{l}\text { Hematological Toxicity } \\
\text { Univariate Analysis }\end{array}$}} & \multirow{2}{*}{\multicolumn{2}{|c|}{ Multivariate Analysis }} & Emesis & - & - & - \\
\hline & & & & & \multicolumn{2}{|c|}{ Univariate Analysis } & \multicolumn{2}{|c|}{ Multivariate Analysis } \\
\hline & OR (95\% Cl) & $P$ & OR (95\% Cl) & $P$ & OR $(95 \% \mathrm{Cl})$ & $P$ & OR (95\% Cl) & $P$ \\
\hline Age & $0.59(0.43-0.82)$ & $0.002^{* *}$ & - & 0.102 & $0.39(0.27-0.55)$ & $<0.001 * * *$ & $0.59(0.37-0.92)$ & $0.020^{*}$ \\
\hline Sex & - & 0.818 & - & - & - & 0.802 & - & - \\
\hline $\mathrm{CCl}$ & - & 0.314 & - & - & - & $0.001^{* * *}$ & - & 0.138 \\
\hline BMI & $0.66(0.43-1.03)$ & $0.062^{*}$ & - & 0.139 & - & 0.126 & - & - \\
\hline $\mathrm{LDH}$ & - & - & - & - & - & - & - & - \\
\hline ALB & - & 0.461 & - & - & - & 0.994 & - & - \\
\hline $\mathrm{Hb}$ & - & - & - & - & - & - & - & - \\
\hline T stage & $1.24(1.02-1.5 I)$ & $0.001^{* * * *}$ & - & 0.072 & $1.62(0.98-2.67)$ & $0.058^{*}$ & - & 0.928 \\
\hline $\mathrm{N}$ stage & $2.12(1.53-2.92)$ & $<0.001 * * *$ & $2.50(1.28-4.88)$ & $0.007 * *$ & $1.69(1.03-2.78)$ & $0.038 *$ & - & 0.348 \\
\hline Clinical stage & $1.67(1.34-2.07)$ & $<0.001 * * *$ & - & 0.944 & $2.42(1.37-4.28)$ & $0.002^{* * * *}$ & - & 0.652 \\
\hline Chemotherapy & $\begin{array}{l}8.27 \\
(4.44-15.42)\end{array}$ & $<0.001 * * *$ & $\begin{array}{l}6.01 \\
(3.11-11.63)\end{array}$ & $<0.00 I^{* * * *}$ & $\begin{array}{l}309.90 \\
(86.20-1|1| 4.20)\end{array}$ & $<0.001 * * *$ & $\begin{array}{l}225.14 \\
(61.91-818.64)\end{array}$ & $<0.001 * * *$ \\
\hline \multirow[t]{2}{*}{ - } & Dermatitis & - & - & - & Mucositis & - & - & - \\
\hline & \multicolumn{2}{|c|}{ Univariate Analysis } & \multicolumn{2}{|c|}{ Multivariate Analysis } & \multicolumn{2}{|c|}{ Univariate Analysis } & \multicolumn{2}{|c|}{ Multivariate Analysis } \\
\hline Characteristics & OR (95\% Cl) & $P$ & OR (95\% Cl) & $P$ & OR(95\% Cl) & $p$ & OR(95\% Cl) & $p$ \\
\hline Age & - & 0.719 & - & - & - & 0.738 & - & - \\
\hline Sex & $0.26(0.06-1.27)$ & $0.053^{*}$ & - & 0.098 & - & 0.780 & - & - \\
\hline $\mathrm{CCl}$ & - & 0.537 & - & - & - & 0.458 & - & - \\
\hline BMI & - & 0.995 & - & - & - & 0.546 & - & - \\
\hline LDH & - & - & - & - & - & - & - & - \\
\hline ALB & $1.12(0.99-1.26)$ & $0.073^{*}$ & - & 0.080 & $1.10(1.03-1.17)$ & $0.005 * *$ & $1.11(1.04-1.19)$ & $0.002 * *$ \\
\hline $\mathrm{Hb}$ & - & - & - & - & - & - & - & - \\
\hline T stage & - & 0.164 & - & - & $2.20(|.3|-3.7 \mid)$ & $0.003 * *$ & - & 0.114 \\
\hline $\mathrm{N}$ stage & - & 0.455 & - & - & $1.84(1.10-3.08)$ & $0.019 *$ & - & 0.134 \\
\hline Clinical stage & - & 0.314 & - & - & $2.13(1.19-3.83)$ & $0.010 * *$ & - & 0.674 \\
\hline Chemotherapy & - & 0.145 & - & - & - & 0.213 & - & - \\
\hline
\end{tabular}

Notes: Variables with a $P<0.10$ in univariable analysis are selected for multivariable analysis; $* P<0.10$ in univariable analysis and $P<0.05$ in multivariable analysis; $* * P<0.01$; $* * * P<0.001$.

Abbreviations: $\mathrm{CCl}$, Charlson comorbidity index; BMI, body mass index; ALB, albumin; LDH, lactic dehydrogenase; Hb, hemoglobin.

outcome was poor for the elderly patients in 2013; and even in 2019, a study still found that the 5-year OS for the elderly stayed low at $50.9 \%{ }^{6}$ In our study, the 5 -year PFS and OS was $66.3 \%$ and $66.8 \%$, also indicating an unsatisfied outcome. The poor outcome of elderly patients was concluded to be related to the degeneration of body functions. Meanwhile, elderly patients were more likely to be graded as advanced stage at diagnosis; $73.4 \%$ of them was staged as stage III or IV in our study. This may be contributed by the high prevalence of comorbidities and the absence of sensitivity, so that the elderly found it hard to notice the self-conscious symptoms of NPC at the early stage, which increased the risk of failure and led to a poor survival outcome. ${ }^{14,15}$ From this point, screening in epidemic area, early diagnosis, and early treatment could provide an effective way for improving treatment outcome. In this study, we also investigated the failure pattern, and the result showed that cancer recurrence and metastasis remained the main cause of death for the elderly patients, accounting for $71.4 \%$, which indicated a positive treatment strategy was required for NPC treatment. Few patients died from comorbidities, consistent with the study from Lin et al. ${ }^{9}$ In this study, some were reported to die from poor nutrient status inducing loss of appetite after treatment, or from intractable epistaxis because of inappropriate care; Some lost life for unexpected reasons such as suicide and apastia, which both were mainly caused by poor psychological condition due to late toxicities. These deaths were a great pity and should be paid attention to. That is, because of the degeneration in 
Table 4 Univariate and Multivariate Analysis of PFS

\begin{tabular}{|l|l|l|l|l|}
\hline \multirow{2}{*}{ Characteristics (No./\%) } & \multicolumn{2}{l|}{ Univariate Analysis } & \multicolumn{2}{l|}{ Multivariate Analysis } \\
\cline { 2 - 5 } & HR (95\% Cl) & P-value & HR (95\% Cl) & P-value \\
\hline Total & - & - & - & - \\
Age & $1.66(1.24-2.22)$ & $0.00 I^{* * *}$ & $1.10(1.05,1.15)$ & $<0.00 I^{* * *}$ \\
Sex & $0.53(0.26-1.07)$ & $0.075^{*}$ & - & - \\
CCl & - & 0.439 & - & - \\
BMI & - & 0.742 & - & - \\
T stage & $1.89(1.12-3.17)$ & $0.014^{*}$ & $1.85(1.10,3.13)$ & $0.022^{*}$ \\
N stage & $1.54(0.95-2.52)$ & $0.083^{*}$ & - & - \\
LDH & $1.00(1.00-1.01)$ & $0.026^{*}$ & - & - \\
ALB & $0.91(0.86-0.97)$ & $0.004^{* *}$ & $0.93(0.88-0.99)$ & $0.019 *$ \\
Hb & - & 0.224 & - & - \\
Treatment & - & 0.173 & - & - \\
\hline
\end{tabular}

Notes: Variables with a $P<0.10$ in univariable analysis are selected for multivariable analysis; $* P<0.10$ in univariable analysis and $P<0.05$ in multivariable analysis; $* * P<0.01 ; * * * P<0.001$.

Abbreviations: PFS, progress-free survival; $\mathrm{CCl}$, Charlson comorbidity index; BMI, body mass index; ALB, albumin; LDH, lactic dehydrogenase; Hb, hemoglobin; RT, radiotherapy.

body function, the elderly sustain poor comprehensive ability, bad memory, unsatisfied operative ability, and fragile psychological condition, they are more vulnerable than the general patients. ${ }^{16}$ Therefore more attention should be paid to both physiological and psychological fragility in the elderly patients; daily life support, nutrition support, and psychological support should be provided at the appropriate time.

Age was considered as a critical role in the survival outcome of the elderly patients of NPC, but the details were out of knowledge. To work out how the age affected the outcome, we divided patients into $60 \leq \mathrm{n}<65$ group, $65 \leq \mathrm{n}<70$ group, and $\geq 70$ group, and a comparison was performed. Although patients in the 60-65 group were not thought to be as "aged" nowadays, they were still ruled out by clinical trials, and we found out that the 5-year PFS and OS of them, which were $74.3 \%$ and $82.7 \%$, were also poorer than the general NPC patients. ${ }^{1}$ The results also indicated that the older a patient was, and the worse the outcome would be, the difference in prognosis of the three groups was of significance. Patients in the over-70-yearold group showed a much poorer outcome with a 5-year

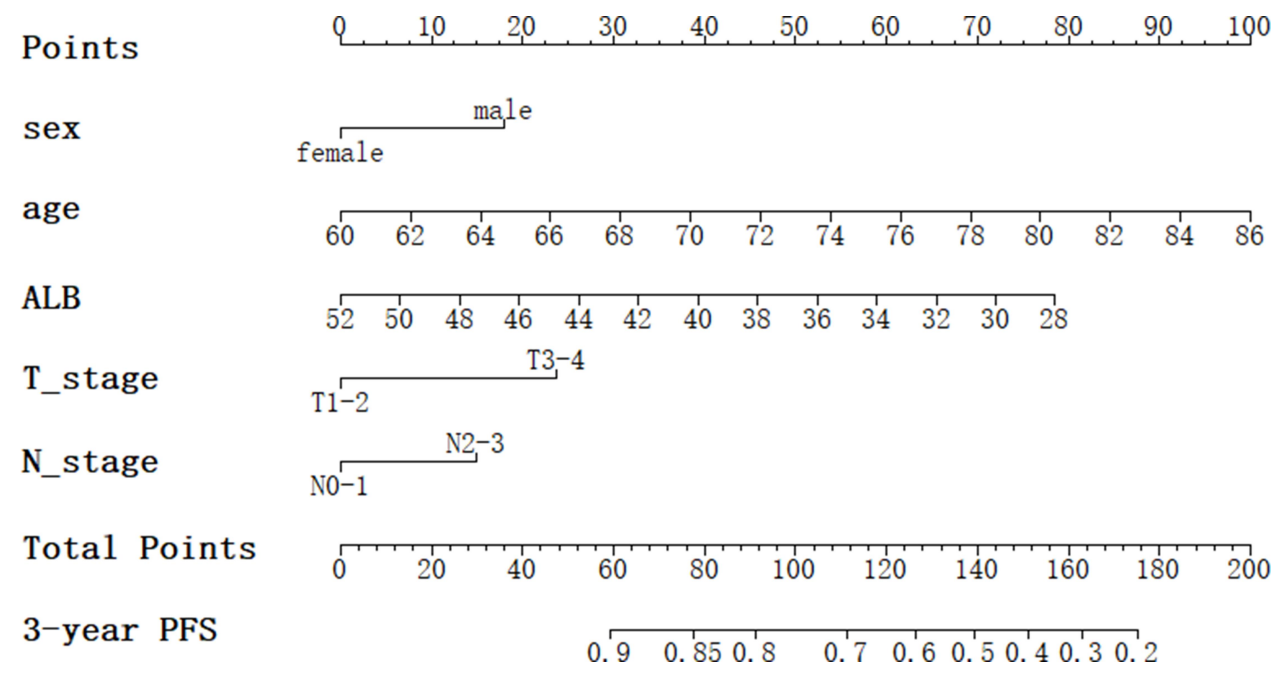

Figure 3 The nomogram for 3-year PFS in the elderly patients with NPC.

Notes: The nomogram is used to predict 3-year PFS for an individual elderly patient. Usage: first, the top line with a name as "points" is corresponding with all variables, draw a straight line up from each variable to the "point" line and get to know the variable's points. Second, sum up points of variables and put the total point at the "total points" line, which is also one-to-to corresponding with the PFS, draw a line down and get to know the 3-year PFS.

Abbreviations: PFS, progress-free survival; NPC, nasopharyngeal carcinoma. 


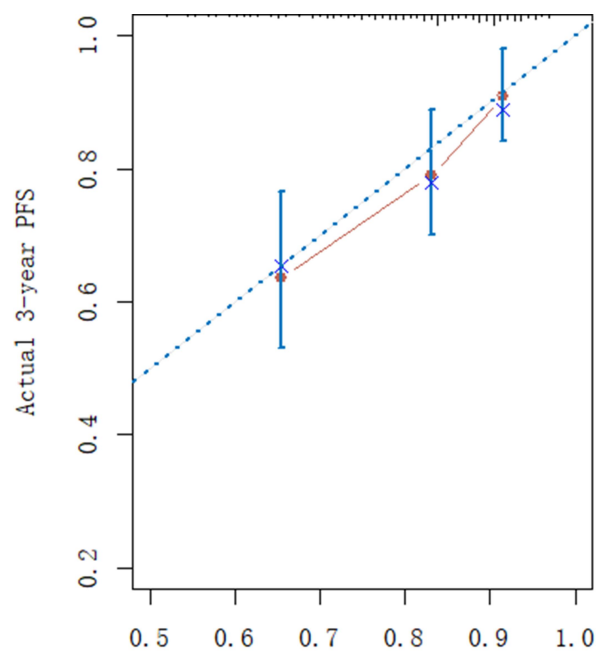

Nomogram-predicted Probility of 3-year PFS

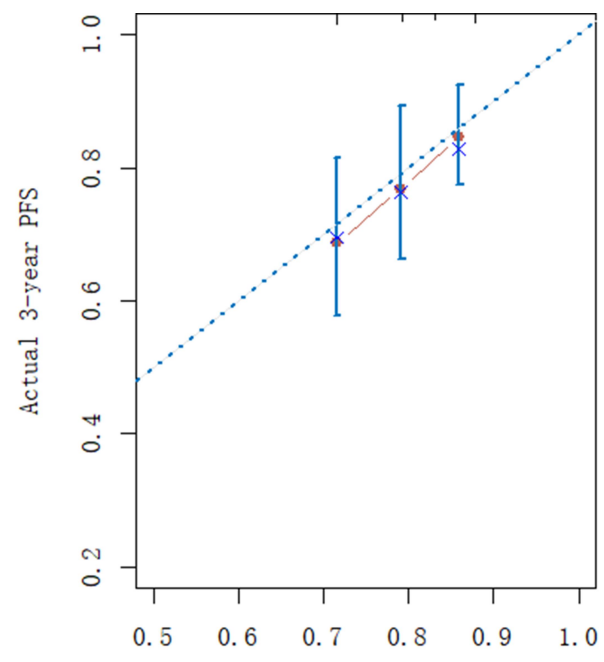

TNM-predicted Probility of 3-year PFS

Figure 4 The calibration plot of nomogram and TNM system in the elderly patients with NPC. Abbreviations: PFS, progress-free survival; NPC, nasopharyngeal carcinoma.

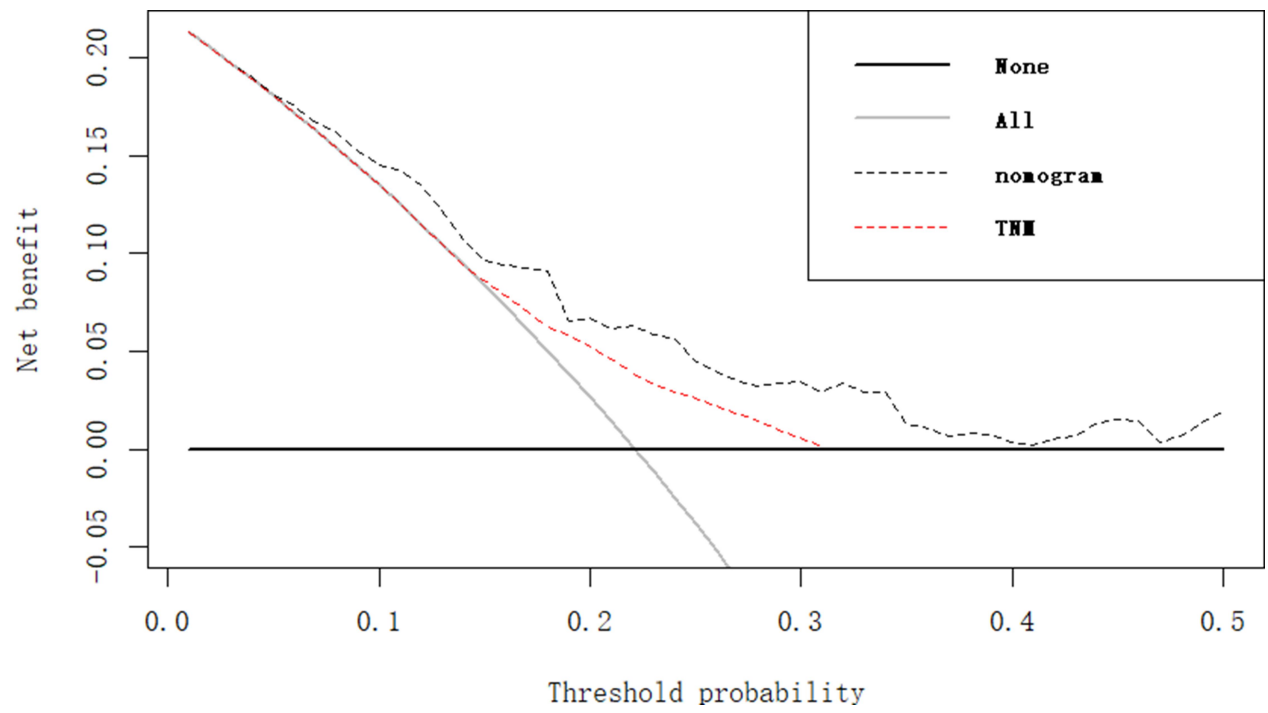

Figure 5 The decision curve of nomogram and TNM system in the elderly patients with NPC.

Notes: The x-axis is determined by the threshold probability, at which the harm of false-positive intervention exceeds the harm of a false-negative non-intervention and thus an intervention is triggered. The $y$-axis is a net benefit. Net benefit is the relative benefit derived from the proportion of true-positive result subtracts the proportion of false-positive result weighted by a ratio from threshold probability. Under the same probability, the clinical usefulness is better when the net benefit is higher.

OS and PFS as $46.5 \%$ and $46.2 \%$, which was in accordance with the study of Lin et al, ${ }^{9}$ revealing that the 5 -year OS of the $<75$ year-old-group was $69 \%$ while the 5 -year OS of the $>75$ year-old-group was $42 \%$. Worse outcome could be explained by the complex physiological and metabolic change of elderly patients, which was not fully understood yet. In the multivariable analysis, age was also proved to have a negative influence on PFS. This underlined the necessary early detection and treatment in elderly patients. Interestingly, regarding acute toxicities, we discovered that elder patients sustained less emesis, which was in accordance with the previous studies; ${ }^{17}$ this is possibly concerned with the decreased gastrointestinal motility due to the degeneration of the body in the elderly. ${ }^{18}$

Numerous studies of multi-center and big sample size reported that chemotherapy benefited the treatment of the general NPC patients, ${ }^{4,19}$ however, there was no certain answer for whether the chemotherapy was safely and effectively feasible for the elderly or not. Some studies 
reported that chemotherapy was tolerable and improved the outcome, ${ }^{20}$ but some argued the opposite. ${ }^{9,21}$ Our study showed that the use of chemotherapy did not gain at all. For the possible cause, there was an assumption that the chemotherapy could improve the outcome in the elderly indeed but it was impeded by the nonstandard regimen and dose, which was common in practice. ${ }^{9}$ Meanwhile, there was another assumption that the elderly were not sensitive to the chemotherapy at all due to their special physiological state. However, this conflict could not be solved under the limitation of the now-existed information, so further research is needed. In terms of acute toxicities, chemotherapy was revealed to be concerned with the hematological toxicity and the degree of emesis. With acute toxicities but without promotion on PFS, chemotherapy should be under careful consideration in the treatment of elderly patients.

Comorbidities have attracted much attention when a clinician is making a strategy of treatment, and its impact can be assessed by the CCI. ${ }^{22} \mathrm{CCI}$ includes common comorbidities from various human systems, such as hypertension, diabetes, heart disease, and immune diseases. The severity of comorbidities is also taken into account, and, according to the impact of comorbidities on the prognosis of diseases, corresponding scores are assigned, ranging from $0-6$ points. A big study of 9300 cases proved that CCI was a meaningful indicator to describe the effect of comorbidities on the prognosis of diseases. ${ }^{23}$ Therefore, CCI was used to quantify the number and degree of comorbidities in this study. The results showed that the comorbidities quantified by CCI were not predictors of the prognosis in elderly NPC patients, which was consistent with the report of Lin et al and $\mathrm{Li}$ et al. ${ }^{6,9}$ And that could be related to the improvement of diagnosis and treatment in modern times, leading to satisfied management of most comorbidities. However, some held contrary opinions that comorbidities were still influential factors indicating poor prognosis. ${ }^{15}$ The contradiction may be partly explained by the limitation of sample size, and the measurement of comorbidities; and it needs to be confirmed by large-scale, multi-center data. On the other hand, comorbidities were often under consideration while a clinician was evaluating the tolerance of the therapy. However, we found that comorbidities were not factors affecting common acute toxicities. Therefore, it is not comorbidities that should be under careful consideration for tolerance, but instead, the inside physiologic reserve, which can be monitored via normal multidisciplinary assessment (echocardiography, liver, and renal test, etc.), should be evaluated. ${ }^{24}$
Nutrition was represented with BMI, ALB, and $\mathrm{Hb}$ in our study. BMI and $\mathrm{Hb}$ were not risk factors, while ALB had a positive influence on prognosis and acute toxicities. ALB was a serological marker indicating nutrition condition, which was proved to be an important biomarker associated with survival of cancer patients. ${ }^{25,26}$ In the elderly patients of NPC, lower ALB decided poorer outcome. Therefore, appropriate nutrition support was in need for better outcome. However, ALB was not helpful in preventing the acute toxicities.

It should be noted that most of the acute toxicities in the elderly patients with NPC were at and under grade II, and no fetal toxicity was observed, which was of consensus with the studies of Lin et $\mathrm{al}^{9,15}$ and Jin et al. This indicated that the acute toxicities during the treatment were completely tolerable, active treatment was possible, and that, even more, the possibility of pushing treatment boundaries is allowed.

We further established a nomogram based on the multivariable analysis of PFS. The nomogram provided a simple and efficient way in predicting the 3-year PFS, and, moreover, it had better performance than the TNM system, and it is hopeful to promote proper and efficient clinical decision-making in the near aging future.

The study had limitations. First, it was a retrospective study, and selective biases inevitably existed, however, it came from the real world and represented the real condition. Second, we performed an internal validation by bootstrapping in the nomogram, however, it would be better if we validated the nomogram externally.

\section{Conclusion}

To sum up, elderly patients with NPC are at the late stage at diagnosed and they sustain a poor prognosis. Tumor recurrence and metastasis were the main reasons for death, meanwhile treatment related deaths caused by poor nutrition status and psychological condition should not be ignored. More attention should be paid to daily life support, nutrition support, and psychological support. Age influenced prognosis negatively, so that early screening in an epidemic area, early detection, and early treatment should be carried out. This indicated that chemotherapy had no impact on the prognosis but the acute toxicities, while comorbidities had no impact on neither the prognosis nor the acute toxicities, however, here exists conflict and more studies are needed. ALB supported better outcome but did not reduce acute toxicities. All the acute toxicities were at and under grade II and tolerable, and 
active treatment could be considered. We further developed an easily applied nomogram, it was superior to the 8th AJCC staging system, and it is hopeful to benefit the decision-making.

\section{Abbreviations}

NPC, nasopharyngeal carcinoma; IMRT, intensitymodulated radiotherapy; TNM system, the American Joint Committee on Cancer/Union for International Cancer Control Tumor-Node-Metastasis staging system; CTCAE, the Common Terminology Criteria for Adverse Events; PTV, planning target volume; GTV, gross tumor volume; CTV, clinical target volume; CCRT, concurrent chemoradiotherapy; IC, induction chemotherapy; AC, adjuvant chemotherapy; PFS, progress-free survival; OS, overall survival; $\mathrm{C}$-index, concordance index; $\mathrm{Hb}$, hemoglobin; ALB, albumin; LDH, lactate dehydrogenase level; CCI, Charlson comorbidity index; BMI, body mass index; RT, radiotherapy; OR, odd ratio; HR, hazard ratio.

\section{Ethical Approval and Consent to Participate}

This study was approved by the Research Ethics Committee of Guangxi Medical University Cancer Hospital (LW2020026), all procedures followed the instruction of the Declaration of Helsinki. Patient consent to review their medical records was not applicable due to the retrospective nature of the study, and the research involved no more than minimal risk to the participants. The data were anonymously analyzed. All the participants' personal information is confidential.

\section{Acknowledgments}

This work was sponsored by The Key Technologies Research and Development Program of Guangxi (AB18221007).

\section{Author Contributions}

All authors made a significant contribution to the work reported, whether that is in the conception, study design, execution, acquisition of data, analysis and interpretation, or in all these areas; took part in drafting, revising, or critically reviewing the article; gave final approval of the version to be published; have agreed on the journal to which the article has been submitted; and agree to be accountable for all aspects of the work.

\section{Disclosure}

The authors report no conflicts of interest in this work.

\section{References}

1. Guo R, Tang -L-L, Mao Y-P, et al. Proposed modifications and incorporation of plasma Epstein-Barr virus DNA improve the TNM staging system for Epstein-Barr virus-related nasopharyngeal carcinoma. Cancer. 2019;125(1):79-89. doi:10.1002/cncr.31741

2. Liang Xin YJ, Ting G, et al. Nasopharynx cancer epidemiology in China. Chin Cancer. 2016;25(11):835-840.

3. Sun Y, Li W-F, Chen N-Y, et al. Induction chemotherapy plus concurrent chemoradiotherapy versus concurrent chemoradiotherapy alone in locoregionally advanced nasopharyngeal carcinoma: a Phase 3, multicentre, randomised controlled trial. Lancet Oncol. 2016;17(11):1509-1520. doi:10.1016/S1470-2045(16)30410-7

4. Zhang Y, Chen $\mathrm{L}, \mathrm{Hu} \mathrm{GQ}$, et al. Gemcitabine and cisplatin induction chemotherapy in nasopharyngeal carcinoma. $N$ Engl J Med. 2019;381 (12):1124-1135. doi:10.1056/NEJMoa1905287

5. Huang Y, Chen W, Haque W, et al. The impact of comorbidity on overall survival in elderly nasopharyngeal carcinoma patients: a National Cancer Data Base analysis. Cancer Med. 2018;7 (4):1093-1101. doi:10.1002/cam4.1377

6. Li Y, Yanfang C, Wu W, Long J, Luo X, Feng J. Clinical characteristics and prognosis of elderly patients with nasopharyngeal carcinoma in intensity - modulated radiation therapy. Chongqing Med Sci. 2019;48

7. Cao C, Hu Q, Chen X. Intensity-modulated radiotherapy for elderly patients with nasopharyngeal carcinoma. Head Neck. 2018;40 (3):590-595. doi:10.1002/hed.25016

8. Gao J, Gao T, Xiaoling F. The prognosis of 134 cases of the elderly nasopharyngeal carcinoma patients. Prat Geriatrics. 2013.

9. Lin J, Han L, Lin S, Rui LI, Yun XU, Pan J. Therapeutic effect of radiotherapy and chemotherapy in 202 elderly patients with nasopharyngeal carcinoma. Chin J Radiat Oncol. 2013;22:461-464.

10. Frank Harrell KL, Mark D. Multivariable prognostic models: issues in developing models, evaluating assumptions and adequacy, and measuring and reducing errors. Stat Med. 1996;15:361-387. doi:10.1002/(SICI)1097-0258(19960229)15:4<361::AID-SIM168>3. $0 . \mathrm{CO} ; 2-4$

11. Vickers AJ, Van Calster B, Steyerberg EW. Net benefit approaches to the evaluation of prediction models, molecular markers, and diagnostic tests. BMJ. 2016;352:i6. doi:10.1136/bmj.i6

12. Ma J, Mai HQ, Hong MH, et al. Results of a prospective randomized trial comparing neoadjuvant chemotherapy plus radiotherapy with radiotherapy alone in patients with locoregionally advanced nasopharyngeal carcinoma. J Clin Oncol. 2001;19(5):1350-1357. doi:10.1200/JCO.2001.19.5.1350

13. Liqin MA, Ling Y, Xiangquan K, Zhang Y, Pan Caizhu XN. The prognosis of 224 cases of elderly patients of nasopharyngeal carcinoma. J Fujian Med Univ. 2007;06:18-20.

14. Xin W, Zhu Yaoguo JW. Clinical observation of 32 elderly patients with nasopharyngeal carcinoma. China Med Pharm. 2013;3(14):144-145+153.

15. Jin YN, Zhang WJ, Cai XY, et al. The characteristics and survival outcomes in patients aged 70 years and older with nasopharyngeal carcinoma in the intensity-modulated radiotherapy era. Cancer Res Treat. 2019;51(1):34-42. doi:10.4143/crt.2017.551

16. Porceddu SV, Haddad RI. Management of elderly patients with locoregionally confined head and neck cancer. Lancet Oncol. 2017;18(5):e274-e283. doi:10.1016/S1470-2045(17)30229-2

17. Singh K, Paul SM, Kober KM, et al. Neuropsychological symptoms and intrusive thoughts are associated with worse trajectories of chemotherapy-induced nausea. J Pain Symptom Manage. 2019. 
18. Le Saux O, Falandry C. Toxicity of cancer therapies in older patients. Curr Oncol Rep. 2018;20(8):64. doi:10.1007/s11912-018-0705-y

19. Chen QY, Wen YF, Guo L, et al. Concurrent chemoradiotherapy vs radiotherapy alone in stage II nasopharyngeal carcinoma: Phase III randomized trial. J Natl Cancer Inst. 2011;103(23):1761-1770. doi:10.1093/jnci/djr432

20. Yang Q, Zhao TT, Qiang MY, et al. Concurrent chemoradiotherapy versus intensity-modulated radiotherapy alone for elderly nasopharyngeal carcinoma patients with pre-treatment Epstein-Barr virus DNA: a Cohort Study in an endemic area with long-term follow-up. $J$ Cancer. 2018;9(17):3023-3031. doi:10.7150/jca.26145

21. Miao J, Wang L, Zhu M, et al. Reprint of long-term survival and late toxicities of elderly nasopharyngeal carcinoma (NPC) patients treated by high-total- and fractionated-dose simultaneous modulated accelerated radiotherapy with or without chemotherapy. Oral Oncol. 2019;90:126-133. doi:10.1016/j.oraloncology.2019.01.005

22. Charlson ME, Pompei P, Ales KL, MacKenzie CR. A new method of classifying prognostic comorbidity in longitudinal studies: development and validation. $J$ Chronic Dis. 1987;40(5):373-383. doi:10.1016/0021-9681(87)90171-8
23. Frenkel WJ, Jongerius EJ, Mandjes-van Uitert MJ, van Munster BC, de Rooij SE. Validation of the Charlson comorbidity index in acutely hospitalized elderly adults: a prospective cohort study. $J$ Am Geriatr Soc. 2014;62(2):342-346. doi:10.1111/jgs. 12635

24. Fiorica F, Zanghi A, Pascale G, et al. Is effective and safe a radiochemotherapy approach in elderly cancer patients? A review. Anticancer Agents Med Chem. 2013;13(9):1430-1437. doi:10.2174/ 18715206113136660354

25. Li X, Qin S, Sun X, et al. Prognostic significance of albumin-globulin score in patients with operable non-small-cell lung cancer. Ann Surg Oncol. 2018;25(12):3647-3659. doi:10.1245/s10434-018-6715-z

26. Salas S, Deville JL, Giorgi R, et al. Nutritional factors as predictors of response to radio-chemotherapy and survival in unresectable squamous head and neck carcinoma. Radiother Oncol. 2008;87 (2):195-200. doi:10.1016/j.radonc.2008.02.011

\section{Publish your work in this journal}

Cancer Management and Research is an international, peer-reviewed open access journal focusing on cancer research and the optimal use of preventative and integrated treatment interventions to achieve improved outcomes, enhanced survival and quality of life for the cancer patient.
The manuscript management system is completely online and includes a very quick and fair peer-review system, which is all easy to use. Visit http://www.dovepress.com/testimonials.php to read real quotes from published authors. 